2. Koller EA, Cross JT, Doraiswamy PM, Malozowski SN. Pancreatitis associated with atypical antipsychotics: from the Food and Drug Administration's MedWatch surveillance system and published reports. Pharmacotherapy. 2003;23(9):1123-30.

3. Potolidis E, Mandros C, Karakitsos D, et al. Quetiapineassociated pancreatitis in a geriatric critical care patient with delirium. Case Rep Psychiatry 2012;2012:625954.

4. Liou LS, Hung YJ, Hsieh $\mathrm{CH}$, et al. Aggravation of hypertriglyceridemia and acute pancreatitis in a bipolar patient treated with quetiapine. Yonsei Med J 2014;55:831-3.

5. Tang HC, Chung KH. Quetiapine-induced neutropenia in a bipolar patient with hepatocellular carcinoma. Int J Psychiatry Med 2014;47:255-61.

\section{Deep vein thrombosis in ulcerative colitis: a case report from Eastern Nepal}

Inflammatory bowel disease (IBD) including ulcerative colitis and Crohn's disease are chronic disorders of unclear pathogenesis. Traditionally considered a relatively uncommon disorder in developing nations like the South Asian countries, its incidencehas been increasingin these areas and awareness regarding the disease is now growing. Extra-intestinal manifestations of IBD are seen to occur in 25 per cent of cases. Vascular manifestations are rare and the most relevant among them are deep veinthrombosis (DVT) and pulmonary embolism (PE). The clinical course is associated with a higher incidence of inflammatory bowel disease, especially among hospitalized patients. ${ }^{1}$

Here we present what we believe to be the first report of a patient of ulcerative colitis with deep vein thrombosis as an extra-intestinal manifestation in a tertiary care center in Nepal.

\section{Case report}

A 65-year old gentleman who was a known case of ulcerative colitis (diagnosed in April 2014) presented to our Emergency department following a general practitioner referral, complaining of a swelling below the left knee, pain and superficial thrombophlebitis. The thrombophlebitis had been present for three days while the swelling and pain were of one day duration. The patient's intestinal disease was clinically in remission as evident by SCCAI $<2$ (Simple Clinical Colitis Activity Index). The patient had been receiving oral mesalamine 2.4 grams once daily fortwo years for ulcerative colitis. He had no known drug allergies.

On examination, he had a hot, swollen and tender swelling below the left knee which was painful to touch on the medial aspect and in the popliteal fossa. The remainder of the examination was normal.

Investigations revealed a raised ESR of $46 \mathrm{~mm} / \mathrm{hr}$, CRP $10.1 \mathrm{mg} / \mathrm{l}$ and D-dimer level of $3000 \mathrm{ng} / \mathrm{ml}$. Venous Doppler of the lower limb revealed a thrombus involving the left external iliac, femoral vein andpopliteal veins with thickened and edematous skin and subcutaneous tissues in the left thigh and leg. The patient also underwent an unprepared sigmoidoscopy for the assessment of disease activity of ulcerative colitis. There was loss of vascular pattern noted in the rectum which was suggestive of mild disease activity). A biopsy was sent to assess the histological disease activity.

The patient was managed with low molecular weight heparin, warfarin and compression stockings for the left leg. He was discharged after 5 days with mesalamine 2.4 grams daily in divided doses and warfarin maintaining the target INR between 2 and 3. He was on regular follow up withPT/INR report on an out-patient basis. He was reassessed with a Doppler ultrasound after 6 months which revealed a thrombus on the posterior wall of the left external iliac vein with no compromise of blood flow distally. He was also on clinical remission with mesalamine. The patient was asked to continue anticoagulation (warfarin) whle this case was being reported. 


\section{Discussion}

Patients with IBD are at an increased risk of thromboembolic complications due to involvement of both the venous and the arterial system. The exact prevalence of thromboembolic complications in UC is debatable due to contrasting studies though it is known that it is a rare extra-intestinal manifestation of ulcerative colitis. In UC, the incidence rate of DVT has been recorded as 30/10,000 person-years. ${ }^{2}$

The exact pathogenetic mechanism of venous thromboembolism in ulcerative colitis is unclear. Fluctuations in the levels of fibrinogen, factors II, V, and VIII , and fibrinopeptide A have all been linked to active IBD. ${ }^{3}$ Our patient was clinically in remission. His sigmoidoscopy revealed a quiescent disease. Thrombosis, vasculitis, and tissue infarction have been proposed as contributing factors in UC and its exacerbations. ${ }^{3}$ The deep vein thrombosis in our patient was unprovoked in nature having no trauma or immobilization apart from other classic precipitating events. There is increased mortality in IBD patients with thromboembolism as an extra-intestinal manifestation.

A recent study showed that IBD patients who experience their first episode of unprovoked VTE have a $33 \%$ risk of a second episode of VTE within 5 years, with a risk of recurrence that is 2.5 -fold higher than that of non- IBD patients after an initial episode of unprovoked VTE. ${ }^{4}$ Among IBD patients who have unprovoked VTE, the benefits of long-term coagulation in reducing recurrent VTE outweigh the risks of the associated bleeding. In particular, extended anticoagulation may be more appropriate for patients who developed VTE in the absence of active disease or other transient provoking factors. ${ }^{5}$ The decision to continue anticoagulation in our patient was made keeping in mind the unprovoked nature of the disease as well as the evidence of residual thrombus in the left external iliac vein when he was reassessed at six months.

Published studiesabout ulcerative colitis are nearly nonexistent in Nepal. To our knowledge, this is the first case of Ulcerative colitis presenting with deep vein thrombosis to be reported from this part of the world.

To conclude, there is increased risk of venous thrombus and thromboembolism in patients with Ulcerative colitis (and Crohn's disease) as compared to the general population. Disease activity might have a significant correlation with the thrombotic/ thomboembolic manifestation; however, it can occur even without any provoking factors. Extended anticoagulation might be recommended for patients who have minimal disease activity or absence of any provoking factor(s).

RABIN SHARMA ${ }^{1}$ MANISH SUBEDI ${ }^{2}$

${ }^{I}$ Department of Gastroenterology

${ }^{2}$ Department of Internal Medicine BPKIHS, Dharan, Nepal

Correspondence: Dr Rabin Sharma Email:docrabinsharma@gmail.com

\section{References}

1. Bernstein $\mathrm{CN}$, Nabalamba A. Hospitalization-based major comorbidity of inflammatory bowel disease in Canada. Can JGastroentero.2007;21:507-11.

2. Bernstein CN, Blanchard JF, Houston DS, Wajda A.The incidence of deep venous thrombosis and pulmonary embolism among patients with inflammatory bowel disease: a population-based cohort study. Thromb Haemost. 2001;85:430-4.

3. Webberly MJ, Hart MT, Melikian V.Thromboembolism in inflammatory bowel disease: role of platelets. Gut.1993;34:247-51.

4. Novacek G,Weltermann A, Sobala A, Tilg H, Petritsch $\mathrm{W}$, Reinisch $\mathrm{W}$ et al. Inflammatory bowel disease is a risk factor for recurrent venous thromboembolism. Gastroenterology 2010; 139:779-87, 787.e1. doi: 10.1053/j. gastro.2010.05.026. Epub 2010 Jun 12

5. Nguyen GC, Bernstein CN. Duration of anticoagulation for the management of venous thromboembolism in inflammatory bowel disease: a decision analysis. Am J Gastroenterol. 2013;108: 1486-95. 